\title{
ACTIVE-PASSIVE MODE-LOCKED AND MECHANICALLY Q-SWITCHED FREQUENCY TUNABLE Nd: GLASS LASER
}

\author{
H. SCHILLINGER ${ }^{1}$ and A. PENZKOFER \\ Naturwissenschaftliche Fakultät II - Physik, Universität Regensburg, D-8400 Regensburg, Fed. Rep. Germany
}

Received 28 December 1987; revised manuscript received 12 April 1988

\begin{abstract}
The performance of an active-passive mode-locked and mechanically Q-switched Nd:glass laser is investigated. The mechanical chopper used for Q-switching reduces the temporal pulse train jitter. The acousto-optic modulator stabilizes the pulse trains. The wavelength tuning from $1048.5 \mathrm{~nm}$ to $1069.5 \mathrm{~nm}$ for a Nd:phosphate glass (Schott LG-703) and from $1056.2 \mathrm{~nm}$ to 1078.3 $\mathrm{nm}$ for a Nd:silicate glass (Schott LG 680) is achieved with a $5 \mathrm{~mm}$ thick single-plate birefringent filter.
\end{abstract}

\section{Introduction}

Passively mode-locked Nd:glass lasers are widely used for the generation of intense picosecond light pulses [1-4]. The shot-to-shot reproducibility of the $\mathrm{Nd}$ : glass lasers has been improved considerably by the combined active and passive mode-locking [512]. Theoretical investigations of the simultaneous active and passive mode-locking are found in refs. [13-15]. Frequency tuning of passively mode-locked $\mathrm{Nd}$ : glass lasers with prisms has been reported (tuning range $1058 \mathrm{~nm}$ to $1066 \mathrm{~nm}$ ) [16].

In this paper we describe a frequency tunable, active-passive mode-locked, and mechanically Qswitched Nd:glass laser. A mechanical chopper defines the onset of laser action (slow Q-switch $[17,18])$ and reduces the jitter in the build-up time of the pulse trains. An acousto-optic modulator $[19,20]$ initiates the mode-locking and improves the shot-to-shot reproducibility. The frequency tuning is achieved with a single-plate birefringent filter [21]. $\mathrm{Nd}$ : silicate glass (Schott LG 680) and Nd:phosphate glass (Schott LG 703) rods are used as active media.

\section{Experimental}

The experimental setup is depicted in fig. 1. The laser oscillator incorporates the laser rod $\mathrm{AM}$, the

1 Present address: Max Planck Institut für Quantenoptik, D-8046 Garching, Fed. Rep. Germany. birefringent filter BRF, and the anti-resonant ring arrangement (Sagnac interferometer) $[23,24]$. Inside the anti-resonant ring the saturable absorber cell SA (passive mode-locking), the acousto-optic modulator (active mode-locking), and the mechanical chopper (active Q-switching) are located.

The saturable absorber Kodak No. 9740 dissolved in 1,2-dichloroethane is used for passive mode-locking. Its absorption recovery time is $\tau_{\mathrm{a}}=11 \mathrm{ps}$ [25] and its saturation intensity is $I_{\mathrm{S}}=2.8 \times 10^{7} \mathrm{~W} / \mathrm{cm}^{2}$ $\left(I_{\mathrm{S}}=h \nu_{\mathrm{L}} / \sigma_{\mathrm{A}} \tau_{\mathrm{A}} ; \sigma_{\mathrm{A}}=6.1 \times 10^{-16} \mathrm{~cm}^{2}[26]\right.$ is the absorption cross-section at the laser frequency $\nu_{\mathrm{L}}$ ). The small signal single-pass transmission through the absorber cell SA is set to $T_{0}=0.81$.

The acousto-optic modulator (Brewster-Brewster cut fused silica, IntraAction model ML-50Q, $50 \mathrm{MHz}$ acoustic frequency, $325 \mathrm{kHz}$ mode-spacing) is placed in the center of the anti-resonant ring. At this position the modulator introduces the smallest losses to the cavity and gives the best pulse shortening (see below fig. 4a). The resonator length is adjusted to the resonance frequency of the acousto-optic modulator (accuracy $\pm 20 \mu \mathrm{m}$ ). The modulator is thermostated to $\pm 1{ }^{\circ} \mathrm{C}$ (temperature drift is $7 \mathrm{kHz} /{ }^{\circ} \mathrm{C}$ [27]).

The chopper wheel rotates at 6000 cycles per minute. The laser beam distance from the rotor axis is $5.5 \mathrm{~cm}$ and the slit moves a distance of $0.5 \mathrm{~mm}$ within $15 \mu \mathrm{s}$. The flashlamp pumping is synchronized with the chopper and laser action starts when the chopper 


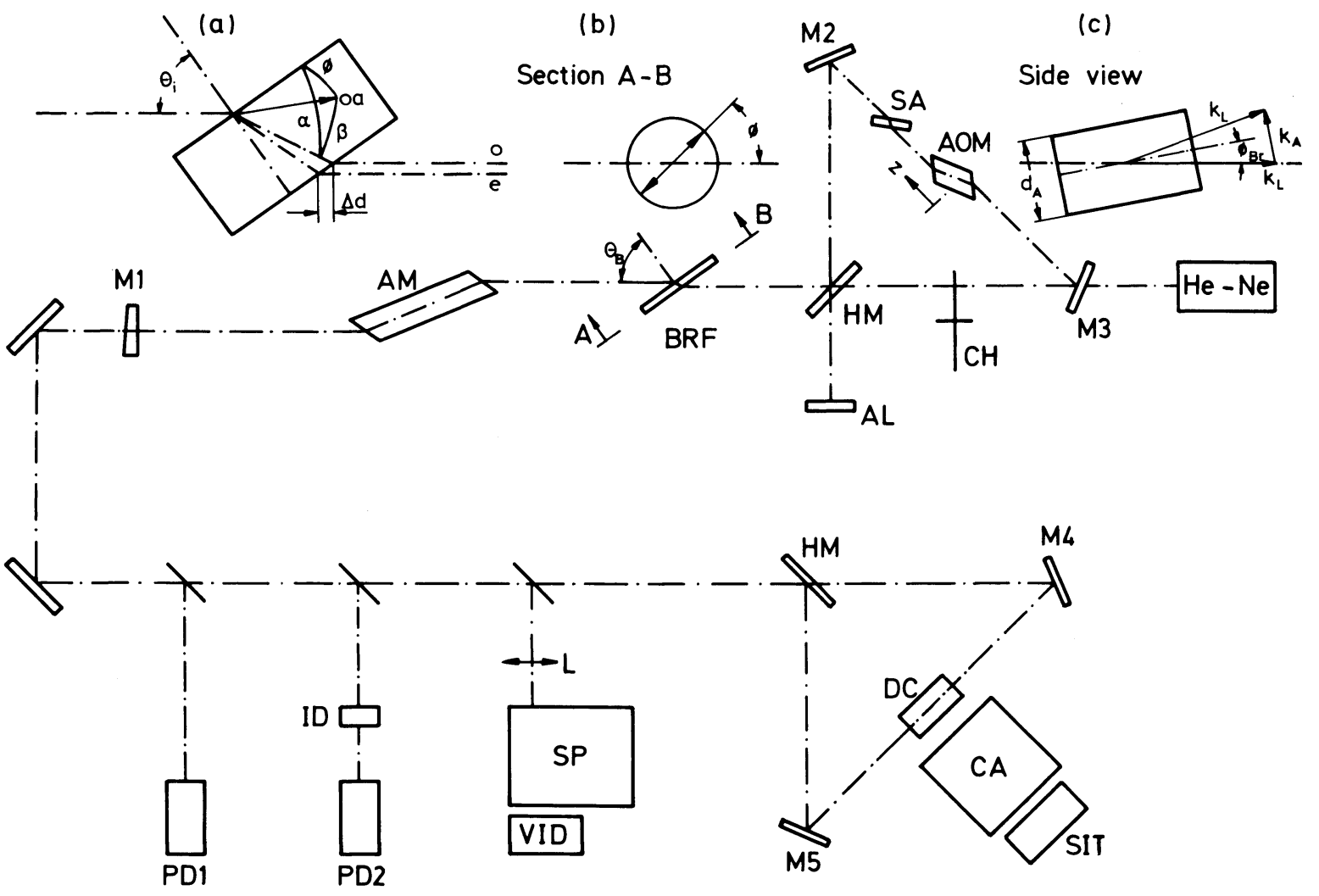

Fig. 1. Experimental setup. M1-M5, dielectric laser mirrors $(\mathrm{R} 1=0.7, \mathrm{R} 2=\mathrm{R} 3=\mathrm{R} 4=\mathrm{R} 5=1.0)$. $\mathrm{HM}, 50 \%$ mirror. AM, active medium, $\mathrm{BRF}$, single-plate birefringent filer. SA, saturable absorber. AOM, acousto-optic modulator. $\mathrm{CH}$, light chopper, He-Ne, He-Ne laser. AL, aluminium mirror for laser alignment. PD1, P D2, photo-detectors. ID, saturable absorber cell for intensity detection. L, lens, SP, grating spectrometer, VID, vidicon. DC, cell containing two-photon fluorescence dye solution. CA, camera. SIT, intensified silicon target vidicon. Inset (a): Light path of ordinary (o) and extra ordinary ray (e) through birefringent filter. oa indicates optical axis of crystal (in A-B plane of inset b). Inset (b): Section A-B through birefringent filter. Inset (c): Illustration of Bragg diffraction in acousto-optic modulator.

wheel opens the laser path; the pulse train maximum appears approximately $10 \mu$ s after full opening of the pathway.

Frequency tuning of the laser is achieved by tuning the azimuthal angle $\phi$ of a single-plate birefringent filter [21] (quartz crystal) which is positioned at the Brewster angle. Two different plate thicknesses of $d_{1}=5 \mathrm{~mm}$ and $d_{2}=10 \mathrm{~mm}$ are used.

A Nd: silicate glass rod (Schott LG 680) and a $\mathrm{Nd}$ :phosphate glass rod (Schott LG 703) are used as active media. In both cases the rod diameter is 9 $\mathrm{mm}$ and the rod length is $13 \mathrm{~cm}$. The doping of the glass rods is 3 weight $\%$ of $\mathrm{Nd}_{2} \mathrm{O}_{3}$.

The laser performance is investigated by pulse train observation (photo-detector PD1), peak intensity detection (energy transmission measurement through Kodak dye No. 9860 in 1,2-dichloroethane [28] with detectors PD1 and PD2), spectrum registration, and pulse duration measurement (two-photon fluorescence technique [29], dye $3 \times 10^{-3}$ molar rhodamine $6 \mathrm{G}$ in methanol).

\section{Results}

The standard operational parameters of the laser together with performance data are listed in table 1.

The normalized stimulated emission cross-section profiles $\sigma_{\mathrm{em}}(\lambda) / \sigma_{\mathrm{em} \text { max }}$ of the active media are depicted in fig. 2 . They have been determined by meas- 
Table 1

Performance data

\begin{tabular}{|c|c|c|c|c|}
\hline Laser glass & LG 680 & & LG 703 & \\
\hline \multicolumn{5}{|l|}{ Standard parameters } \\
\hline Flashlamp voltage [V] & 1380 & & 1380 & \\
\hline Modulator power $P_{\mathrm{A}}[\mathrm{W}]$ & 4 & & 4 & \\
\hline AOM frequency $\nu_{\mathrm{A}}[\mathrm{MHz}]$ & 48.75 & & 48.75 & \\
\hline Modulator position $z[\mathrm{~cm}]$ & 0 & & 0 & \\
\hline Absorber transmission $T_{0}$ & 0.81 & & 0.81 & \\
\hline Laser wavelength $\lambda_{\mathrm{L}}[\mathrm{nm}]$ & 1060 & & 1053.6 & \\
\hline BRF thickness $t[\mathrm{~mm}]$ & 5 & 10 & 5 & 10 \\
\hline \multicolumn{5}{|l|}{ Results } \\
\hline Tuning range $[\mathrm{nm}]$ & $\begin{array}{c}1056.2-1078.3 \\
8 \pm 1\end{array}$ & $1056.8-1067.6$ & $1048.5-1069.5$ & $\begin{array}{c}1050.3-1062.0 \\
10 \pm 2\end{array}$ \\
\hline$\Delta t_{\mathrm{L}}[\mathrm{ps}]$ & $10^{9}$ & & & $10^{9}$ \\
\hline$I_{\mathrm{OL}}{ }^{\text {a) }}\left[\mathrm{W} / \mathrm{cm}^{2}\right]$ & 500 & 500 & 500 & 500 \\
\hline Pulse train width ${ }^{\mathrm{b})}$ [ns] & \pm 300 & \pm 300 & \pm 300 & \pm 300 \\
\hline Jitter $[\mathrm{ns}]$ & & & & \\
\hline
\end{tabular}

a) At pulse train maximum outside output coupler M1. ${ }^{\text {b) }}$ FWHM.

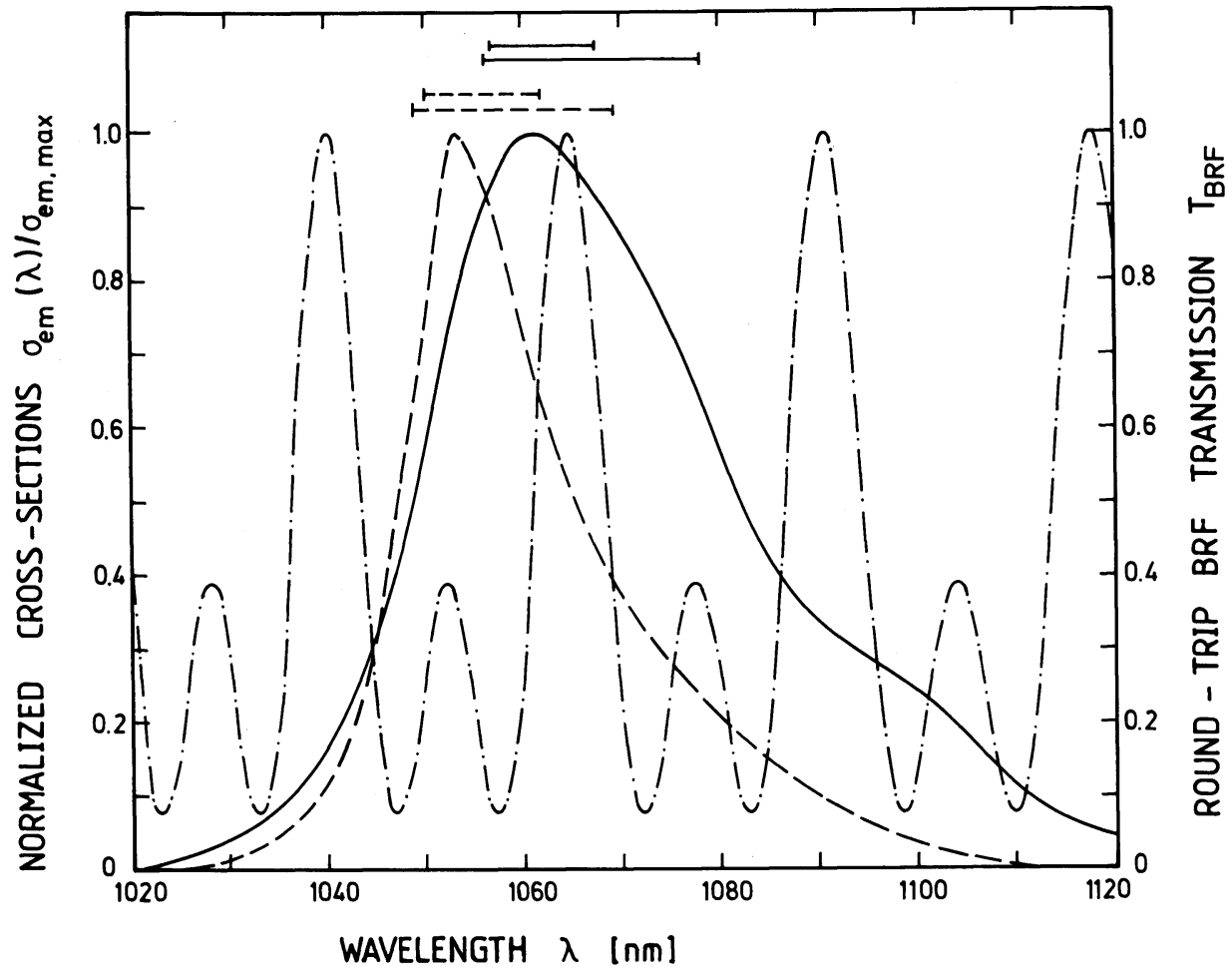

Fig. 2. Stimulated emission cross-section spectra of $\mathrm{Nd}$ : glasses and round-trip transmission of a single-plate birefringent filter. Solid curve, Nd: silicate glass Schott LG 680. Dashed curve, Nd:phosphate glass Schott LG 703. Dash-dotted curve, round-trip transmission through $5 \mathrm{~mm}$ thick birefringent filter. Horizontal bars indicate wavelength tuning regions obtained with $5 \mathrm{~mm}$ (long bars) and $10 \mathrm{~mm}$ (shot bars) birefringent filters. 
uring the fluorescence spectra $E(\lambda)$ with a selfassembled spectro-fluorometer [30] $\quad\left(\sigma_{\mathrm{em}}(\lambda) \propto \lambda^{4}\right.$ $E(\lambda)[31])$. The absolute stimulated emission crosssections are $\sigma_{\text {em.max }}=2.9 \times 10^{-20} \mathrm{~cm}^{2}$ for LG 680 and $4.1 \times 10^{-20} \mathrm{~cm}^{2}$ for LG 703 [32].

The laser wavelength is tuned by rotation of the birefringent filter BRF (azimuthal angle $\phi$ ). The variation of the laser wavelength versus tuning angle $\phi$ is depicted in fig. $3 \mathrm{~b}$ for the $\mathrm{Nd}$ : silicate glass $\mathrm{LG}$ 703 and the $5 \mathrm{~mm}$ thick birefringent filter (circles and dashed curve). The corresponding laser threshold versus angle $\phi$ is depicted in fig. $3 \mathrm{a}$ ( $500 \mu \mathrm{F}$ capacity of condenser of power supply). The obtained tuning range is $21 \mathrm{~nm}$ (from $1048.5 \mathrm{~nm}$ to 1069.5 $\mathrm{nm})$. The tuning ranges of LG 703 and LG 680 for

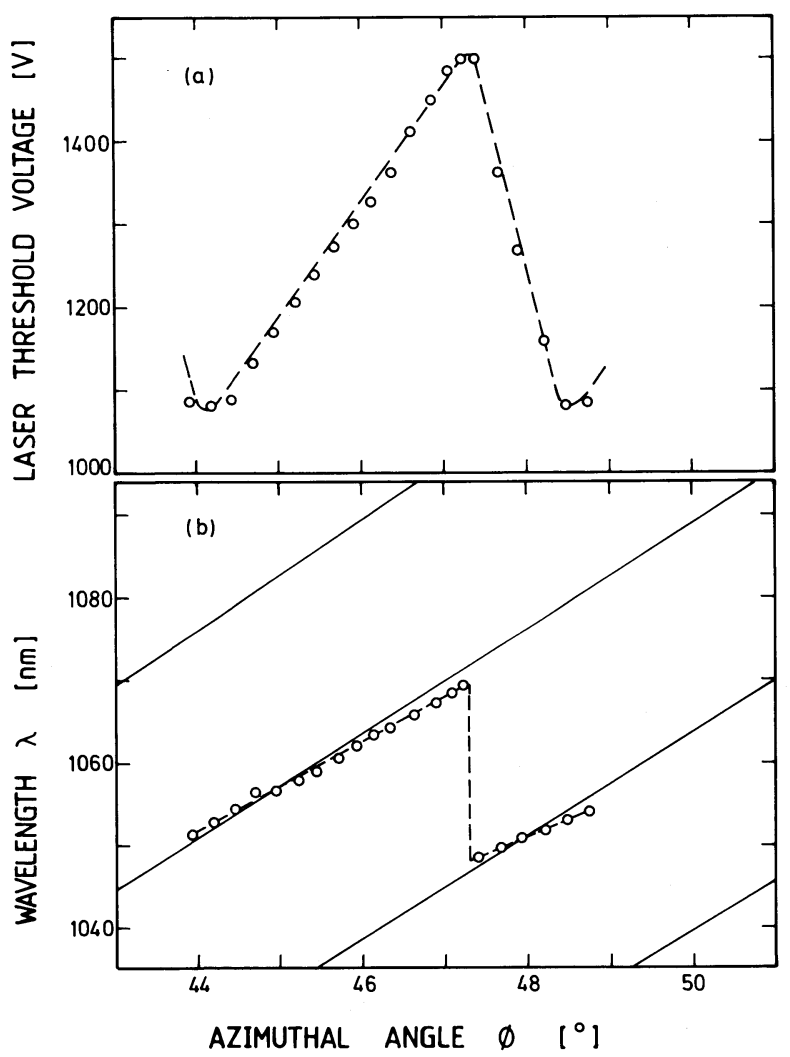

Fig. 3. Laser wavelengths (b) and laser threshold voltage (a) versus azimuthnal angle $\rho$ of a $5 \mathrm{~mm}$ thick birefringent filter. Circles belong to Nd:phosphate glass (Schott LG 703). The solid curves (b) are calculated $\left(\delta_{\mathrm{c}}-\delta_{\mathrm{o}}=2 m\right.$, upper curve $m=41$, lower curve $m=44)$. The dashed curves connect the experimental points. the birefringent filter thicknesses of $5 \mathrm{~mm}$ and $10 \mathrm{~mm}$ are collected in table 1 .

The pulse durations of the hybridly mode-locked laser are 8 to $10 \mathrm{ps}$ (see table 1 ). The pulse train widths (fwhm) are approximately $500 \mathrm{~ns}$, and the pulse train widths at one tenth height are roughly 1 $\mu \mathrm{s}$.

The mechanical chopper reduces the time jitter of the pulse train maximum. Without the chopper, the pulse train maximum appears approximately $500 \mu \mathrm{s}$ after firing the flashlamps and the time jitter is approximately $15 \mu \mathrm{s}$. With the mechanical chopper the pulse train maximum appears approximately $25 \mu \mathrm{s}$ after the beginning of opening the light path and the jitter is approximately $300 \mathrm{~ns}$.

\section{Discussion}

In the following the experimental results are compared with predictions of the birefringent filter theory (frequency tuning) and the acousto-optic modulator theory (active mode-locking).

\subsection{Frequency tuning with birefringent filter}

The theory of birefringent filters is given in ref. [21 ]. The action of the single-plate birefringent applied in our laser arrangement is discussed here.

Starting with p-polarized light $\boldsymbol{E}_{\mathrm{i}}=\left(\begin{array}{l}1 \\ 0\end{array}\right) E_{\mathrm{i}}$ between $\mathrm{HM}$ and BRF of fig. 1 the round-trip transmission $T_{\mathrm{BRF}}$ is given by

$T_{\mathrm{BRF}}=\left|\boldsymbol{E}_{\mathrm{o}}\right|^{2} /\left|\boldsymbol{E}_{\mathrm{i}}\right|^{2}$,

with

$E_{0}=M_{\mathrm{ARR}} \mathbf{M}_{\mathrm{SA}} \mathbf{M}_{\mathrm{AOM}} \mathbf{M}_{\mathrm{BRF}} \mathbf{M}_{\mathrm{LR}} \mathbf{M}_{\mathrm{M} 1} \mathbf{M}_{\mathrm{LR}} \mathbf{M}_{\mathrm{BRF}} E_{\mathrm{i}}$,

The Ms represent $2 \times 2$ Jones matrices [33]. The birefringent filter matrix is given by [21]

$$
\begin{aligned}
& M_{11, \mathrm{BRF}}=(1 / A)\left[\exp \left(\mathrm{i} \delta_{\mathrm{e}}\right)\left(n_{\mathrm{o}}^{2}-\sin ^{2} \theta_{\mathrm{i}}\right) \cos ^{2} \phi\right. \\
& \left.\quad+\exp \left(\mathrm{i} \delta_{\mathrm{o}}\right) n_{\mathrm{o}}^{2} \sin ^{2} \phi\right], \\
& M_{12, \mathrm{BRF}}=\left(q_{\mathrm{BRF}} / A\right)\left[\exp \left(\mathrm{i} \delta_{\mathrm{e}}\right)-\exp \left(\mathrm{i} \delta_{\mathrm{o}}\right)\right] \\
& \quad \times n_{\mathrm{o}} \sin \phi \cos \phi\left(n_{\mathrm{o}}^{2} \sin ^{2} \theta_{\mathrm{i}}\right)^{1 / 2}, \\
& M_{21, \mathrm{BRF}}=M_{12, \mathrm{BRF}},
\end{aligned}
$$




$$
\begin{gathered}
M_{22, \mathrm{BRF}}=\left(q_{\mathrm{BRF}}^{2} / A\right)\left[\exp \left(\mathrm{i} \delta_{\mathrm{e}}\right) n_{\mathrm{o}}^{2} \sin ^{2} \phi\right. \\
\left.+\exp \left(\mathrm{i} \delta_{\mathrm{o}}\right)\left(n_{\mathrm{o}}^{2}-\sin ^{2} \theta_{\mathrm{i}}\right) \cos ^{2} \phi\right] .
\end{gathered}
$$

The parameters in eqs. $(3 a)-(3 d)$ are the extra-ordinary phase

$$
\delta_{\mathrm{e}}=(2 \pi / \lambda)\left[n_{\mathrm{e}}\left(\beta_{\mathrm{e}}\right) d_{\mathrm{e}}+\Delta d\right] \text {, }
$$

the ordinary phase

$$
\delta_{\mathrm{o}}=(2 \pi / \lambda) n_{\mathrm{o}} d_{\mathrm{o}},
$$

the extra-ordinary refractive index

$$
n_{\mathrm{e}}\left(\beta_{\mathrm{e}}\right)=\left[\left(\cos ^{2} \beta_{\mathrm{e}}\right) / n_{\mathrm{o}}^{2}+\left(1-\cos ^{2} \beta_{\mathrm{e}}\right) / n_{\mathrm{e}}^{2}\right]^{-1 / 2},
$$

the extra-ordinary path length

$d_{\mathrm{e}}=t\left[1-\sin ^{2} \theta_{\mathrm{i}} / n_{\mathrm{e}}^{2}\left(\beta_{\mathrm{e}}\right)\right]^{-1 / 2}$,

and the ordinary path length

$d_{\mathrm{o}}=t\left[1-\sin ^{2} \theta_{\mathrm{i}} / n_{\mathrm{o}}^{2}\right]^{-1 / 2}$.

The angle $\beta_{\mathrm{e}}$ (inset a of fig. 1) is derived by use of the relations $\cos \beta_{\mathrm{e}}=\cos \phi \cos \alpha_{\mathrm{e}}$ (spherical trigonometry) and $\sin \theta_{\mathrm{i}}=n_{\mathrm{e}}\left(\beta_{\mathrm{e}}\right) \cos \alpha_{\mathrm{e}}$ (Snell's law). It is

$$
\begin{aligned}
\beta_{\mathrm{e}} & =\arccos \left\{n_{\mathrm{e}}^{-1} \cos \phi \sin \theta_{\mathrm{i}}\right. \\
& \left.\times\left[1-\cos ^{2} \phi \sin ^{2} \theta_{\mathrm{i}}\left(1 / n_{\mathrm{o}}^{2}-1 / n_{\mathrm{e}}^{2}\right)\right]^{-1 / 2}\right\} .
\end{aligned}
$$

The additional path-length $\Delta d$ of the extra-ordinary ray (inset a of fig. 1) is

$\Delta d=t \sin \theta_{\mathrm{i}}\left[\tan \left(\pi / 2-\alpha_{\mathrm{o}}\right)-\tan \left(\pi / 2-\alpha_{\mathrm{e}}\right)\right]$,

$\left(\alpha_{\mathrm{o}}=\arccos \left(n_{\mathrm{o}}^{-1} \sin \theta_{\mathrm{i}}\right), \quad \alpha_{\mathrm{e}}=\arccos \left[n_{\mathrm{e}}^{-1}\left(\beta_{\mathrm{e}}\right)\right.\right.$ $\left.\sin \left(\theta_{\mathrm{i}}\right)\right]$ from Snell's law $) . q$ is the transmission factor of s-polarized light through a single surface. It is

$q=1-r_{\mathrm{s}}^{2}=1-\left(\frac{\cos \theta_{\mathrm{i}}-\left(n^{2}-\sin ^{2} \theta_{\mathrm{i}}\right)^{1 / 2}}{\cos \theta_{\mathrm{i}}+\left(n^{2}-\sin ^{2} \theta_{\mathrm{i}}\right)^{1 / 2}}\right)^{2}$.

For the Brewster angle $\left[\theta_{\mathrm{i}}=\theta_{\mathrm{B}}=\arctan (n)\right] q$ reduces to

$q=2 n /\left(1+n^{2}\right)$.

$n_{\mathrm{o}}=1.534292$ and $n_{\mathrm{e}}=1.543039$ are the ordinary and extra-ordinary principle refractive indices of the quartz crystal at $1053 \mathrm{~nm}$ [34]. $t$ is the thickness of the birefringent filter. The abbreviation $A$ is given by $A=n_{\mathrm{o}}^{2}-\cos ^{2} \phi \sin ^{2} \theta_{\mathrm{i}}$.

The other Jones matrices $M_{\mathrm{LR}}$ (laser rod), $M_{\mathrm{M} 1}$ (output coupler), $M_{\mathrm{AOM}}$ (acousto-optic modulator), $M_{\mathrm{SA}}$ (saturable absorber cell), and $M_{\mathrm{ARR}}$ (antiresonant ring) are diagonal $\left(M_{12}=M_{21}=0\right)$ with $M_{11, \mathrm{LR}}=M_{11, \mathrm{AOM}}=M_{11, \mathrm{SA}}=M_{11, \mathrm{ARR}}=1$,

$M_{11, \mathrm{M} 1}=-1, \quad M_{22, \mathrm{LR}}=q_{\mathrm{LR}}^{2}, \quad M_{22, \mathrm{M} 1}=-1$, $M_{22, \mathrm{AOM}}=q_{\mathrm{AOM}}^{2}, \quad M_{22, \mathrm{SA}}=q_{\mathrm{SA}}^{2}, \quad$ and $\quad M_{22, \mathrm{ARR}}=$ $R_{\mathrm{s}} T_{\mathrm{s}} /\left(R_{\mathrm{p}} T_{\mathrm{p}}\right)$. The $q$ values are given by eq. $(4 \mathrm{~h})$ or (4i) with the appropriate refractive indices. $R_{\mathrm{s}}, R_{\mathrm{p}}$ and $T_{\mathrm{s}}, T_{\mathrm{p}}$ are the reflectivities and transmissions of the anti-resonant ring mirror HM for perpendicular and parallel polarized light (in our case $R_{\mathrm{p}}=T_{\mathrm{p}}=0.5$, $\left.R_{\mathrm{s}}=0.905, T_{\mathrm{s}}=0.095\right)$.

In fig. 2 the calculated round-trip transmission of the $5 \mathrm{~mm}$ thick birefringent filter is included for the azimuthal angle $\phi=46.204^{\circ}$. The wavelengths of peak transmission ( $T_{\mathrm{BRF}}=1, \delta_{\mathrm{e}}-\delta_{\mathrm{o}}=2 \pi m, m$ integer $)$ versus angle $\phi$ are depicted in fig. 3b. The free spectral range of the single plate birefringent filters (wavelength difference between two transmission maxima) is $25 \mathrm{~nm}$ for $t=5 \mathrm{~mm}$ and $12.5 \mathrm{~nm}$ for $t=10 \mathrm{~mm}$.

The laser operates at the wavelength $\lambda_{\mathrm{L}}$ where the net gain

$G_{\text {net }}\left(\lambda_{\mathrm{L}}\right)=T_{\mathrm{BRF}}\left(\lambda_{\mathrm{L}}\right) T_{\mathrm{ARR}} T_{\mathrm{SA}} R_{\mathrm{M} 1} G\left(\lambda_{\mathrm{L}}\right)$,

is maximal. $R_{\mathrm{M} 1}$ is the reflectivity of the output mirror M1. $T_{\mathrm{ARR}}$ is the transmisison through the antiresonant ring including the acousto-optic modulator (see eqs. (8) and (9)). $T_{\mathrm{SA}}$ is the transmission through the saturable absorber.

$G(\lambda)=G_{0}^{\left[\sigma_{\mathrm{em}}(\lambda) / \sigma_{\mathrm{em} . \max }\right]}$,

is the gain factor of the active medium. $G_{0}$ is the peak gain at $\lambda_{\max }$. The wavelength dependence of the gain, $G(\lambda)$, reduces the experimental tuning range to approximately 80 percent of the free spectral range of the birefringent filter (see experimental points in fig. $3 b)$. 


\subsection{Active mode-locking with acousto-optic modulator}

In the cousto-optic modulator the light beam is diffracted at the acoustic standing wave grating (Bragg mode of operation [19]). The modulator cell is tilted to the Bragg angle $\phi_{\mathrm{Br}}$ for optimum light diffraction. This angle is given by (inset $\mathrm{c}$ of fig. 1):

$\phi_{\mathrm{Br}} \approx \sin \phi_{\mathrm{Br}}=\frac{k_{\mathrm{A}}}{2 k_{\mathrm{L}}}=\frac{\nu_{\mathrm{A}} c_{0}}{2 v_{\mathrm{A}} \nu_{\mathrm{L}} n_{\mathrm{L}}}=\frac{\lambda_{\mathrm{L}}}{2 \lambda_{\mathrm{A}}}$,

$k_{\mathrm{A}}=2 \pi \nu_{\mathrm{A}} / v_{\mathrm{A}}$ is the acoustic wave vector, $\nu_{\mathrm{A}}$ the acoustic frequency, and $v_{\mathrm{A}}$ the acoustic velocity $\left(v_{\mathrm{A}} \approx 5.95 \times 10^{3} \mathrm{~m} / \mathrm{s}[19]\right) . k_{\mathrm{L}}=2 \pi \nu_{\mathrm{L}} n_{\mathrm{L}} / c_{0}$ is the laser wave vector, $\nu_{\mathrm{L}}$ is the laser frequency, $n_{\mathrm{L}}$ the refractive index and $c_{0}$ the vacuum light velocity. The standing wave condition requires $d_{\mathrm{A}}=m \lambda_{\mathrm{A}} / 2$ where $m$ is an integer and $d_{\mathrm{A}}$ is the modulator thickness. $d_{\mathrm{A}}$ may be slightly varied by temperature tuning. The resonator round-trip time $t_{\mathrm{R}}$ has to be adjusted to the acoustic frequency by $t_{\mathrm{R}}=\left(2 \nu_{\mathrm{A}}\right)^{-1}$.

The light transmission (undeflected beam) through the acousto-optic modulator oriented under Brewster angle is [18]

$T_{\mathrm{AOM}}(t)=\cos ^{2}\left[\delta_{\mathrm{M}} \sin \left(2 \pi \nu_{\mathrm{A}} t\right)\right]$,

$\delta_{\mathrm{M}}$ is the modulation index. It is proportional to the square root of the RF drive power $P_{\mathrm{A}}$ and is inverse proportional to the laser wavelength $\lambda_{\mathrm{L}}$, i.e. $\delta_{\mathrm{M}}=$ $\kappa P_{\mathrm{A}}^{1 / 2} / \lambda_{\mathrm{L}}$. For $\delta_{\mathrm{M}}=\delta_{\mathrm{M}, 1 / 2}=1.27$ the time averaged transmission

$\bar{T}_{\mathrm{AOM}}=\nu_{\mathrm{A}} \int_{0}^{\nu_{\mathrm{A}}^{-1}} \cos ^{2}\left[\delta_{\mathrm{M}} \sin \left(2 \pi \nu_{\mathrm{A}} t^{\prime}\right)\right] \mathrm{d} t^{\prime}$,

is equal to 0.5. According to ref. [27] the corresponding drive power is $P_{\mathrm{A}, 1 / 2} \approx 2 \mathrm{~W}$ at $\lambda_{\mathrm{L}}=1.06 \mu \mathrm{m}$.

If the acousto-optic modulator is positioned a distance $z$ from the center of the antiresonant ring, the output transmission of the ring is [22]

$$
\begin{aligned}
& T_{\mathrm{ARR}}(t, z)=\mid \rho_{\mathrm{p}} \tau_{\mathrm{p}} t_{\mathrm{AOM}}\left(t-z / c_{0}\right) \\
& +\left.\rho_{\mathrm{p}} \tau_{\mathrm{p}} t_{\mathrm{AOM}}\left(t+z / c_{0}\right)\right|^{2},
\end{aligned}
$$

where $\rho_{\mathrm{p}}^{2}=R_{\mathrm{p}}$ is the reflectivity of the mirror $\mathrm{HM}$ for parallel polarized light, $\tau_{\mathrm{p}}^{2}=1-R_{\mathrm{p}}$ is the transmission through the mirror $\mathrm{HM}$, and $t_{\mathrm{AOM}}^{2}=T_{\mathrm{AOM}}$ is the transmission through the acousto-optic modulator. Insertion of eq. (7) gives

$$
\begin{aligned}
& T_{\mathrm{ARR}}(t, z) \\
& \quad=R_{\mathrm{p}}\left(1-R_{\mathrm{p}}\right)\left\{\cos \left[\sigma_{\mathrm{M}} \sin \left(2 \pi \nu_{\mathrm{A}}\left(t-z / c_{0}\right)\right)\right]\right. \\
& \left.\quad+\cos \left[\delta_{\mathrm{M}} \sin \left(2 \pi \nu_{\mathrm{A}}\left(t+z / c_{0}\right)\right)\right]\right\}^{2} .
\end{aligned}
$$

For $z=0$ (center of antiresonant ring) eq. (8) reduces to

$T_{\text {ARR }}(t, 0)=R_{\mathrm{p}}\left(1-R_{\mathrm{p}}\right) \cos ^{2}\left[\delta_{\mathrm{M}} \sin \left(2 \pi \nu_{\mathrm{A}} t\right)\right]$.

In fig. 4a the transmissions $T_{\mathrm{ARR}}(t, z)$ are shown for
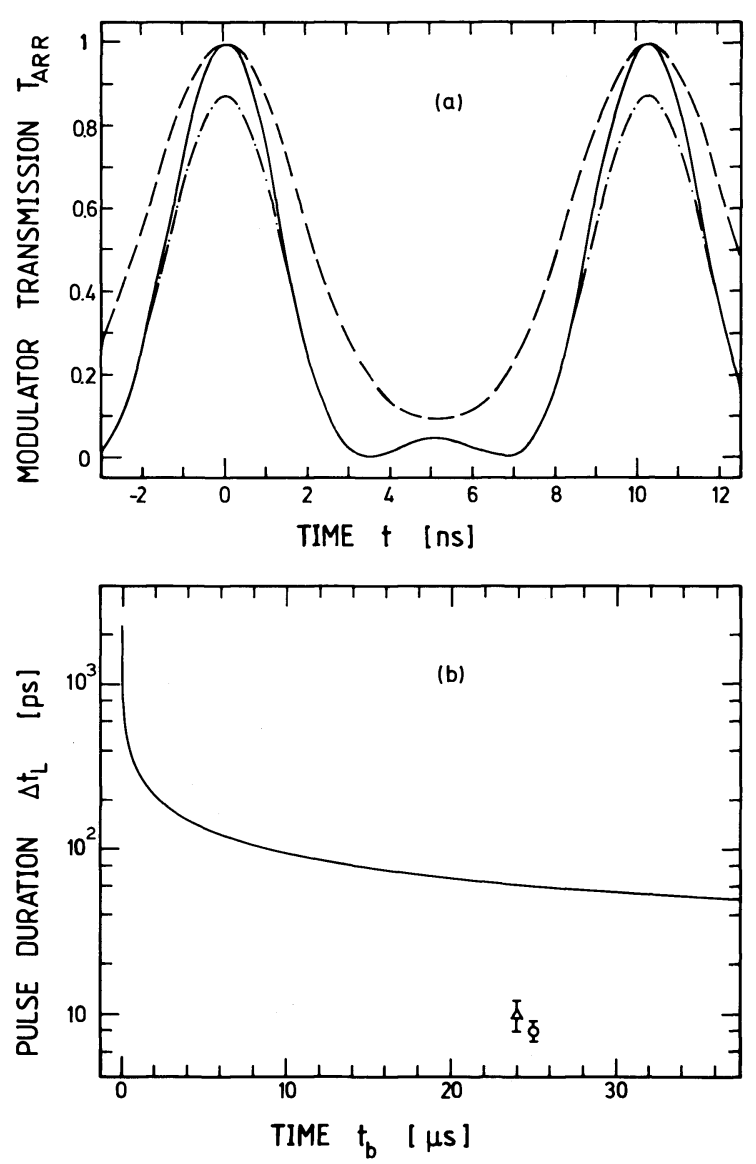

Fig. 4. (a) Transmission through antiresonant ring due to acoustooptic modulator. Dashed curve, acousto-optic modulator in center of antiresonant ring $(z=0)$ and modulator power $P_{\mathrm{A}}=2 \mathrm{~W}$. Solid curve, $z=0 \mathrm{~cm}$ and $P_{\mathrm{A}}=4 \mathrm{~W}$. Dash-dotted curve, $z=20 \mathrm{~cm}$ and $P_{\mathrm{A}}=4 \mathrm{~W}$. (b) Pulse duration $\Delta t_{\mathrm{L}}$ versus build-up time $t_{\mathrm{b}}$ (number of round-trips $m_{\mathrm{R}}=t_{\mathrm{b}} / t_{\mathrm{R}} ; t_{\mathrm{R}}$ resonator round-trip time). Curve is calculated (see text). The experimental points belong to the hybridly mode-locked laser. Circle, LG 680 and birefringent filter thickness $t=5 \mathrm{~mm}$. Triangle, LG 703 and $t=10 \mathrm{~mm}$. 
(i) $P_{\mathrm{A}}=P_{\mathrm{A}, 1 / 2}=2 \mathrm{~W}, z=0, R_{\mathrm{p}}=0.5$ (dashed curve), (ii) $P_{\mathrm{A}}=4 \mathrm{~W}, z=0, R_{\mathrm{p}}=0.5$ (solid curve), and (iii) $P_{\mathrm{A}}=4 \mathrm{~W}, z=20 \mathrm{~cm}, R_{\mathrm{p}}=0.5$ (dash-dotted curve).

A lower limit of the pulse duration of the actively mode-locked laser after $m_{\mathrm{R}}$ round-trips may be estimated by the relations $T_{\mathrm{ARR}}^{m_{R}}\left(t_{1 / 2}\right)=0.5$ and $\Delta t_{\mathrm{L}}=2 t_{1 / 2} \quad(\mathrm{fwhm})$. The calculated pulse duration $\Delta t_{\mathrm{L}}$ versus build-up time $t_{\mathrm{b}}=m_{\mathrm{R}} t_{\mathrm{R}}$ is plotted in fig. $4 \mathrm{~b}$ (solid curve). The experimental pulse durations of the hybridly mode-locked laser are included in fig. $4 \mathrm{~b}$. The pulse shortening action of the saturable absorber is seen clearly.

\section{Conclusions}

The frequency tunable, mechanically Q-switched, and hybridly mode-locked $\mathrm{Nd}$ : glass laser described allows a wavelength tuning of approximately $20 \mathrm{~nm}$ around the center lasing wavelength. The shot-to-shot reproducibility is increased by the acousto-optic modulator. The temporal jitter of the pulse train maximum could be reduced to \pm 300 ns. A further reduction of the jitter is expected by using a fast electrooptical Q-switch instead of the slow mechanical Q-switch.

\section{Acknowledgements}

The authors thank Th. Ascherl for technical assistance. They thank the Rechenzentrum of the University for disposal of computer time.

\section{References}

[1 ] D.J. Bradley, in: Ultrafast light pulses, ed. S.L. Shapiro (Springer-Verlag, Berlin, 1977) p. 18.

[2] D. von der Linde, Appl. Phys. 2 (1973) 281.

[3] A. Laubereau and W. Kaiser, Opto-Electron. 6 (1974) 1.
[4] A. Penzkofer and F. Graf, Opt. Quant. Electron. 17 (1985) 219.

[5] S. Kishida and T. Yamane, Optics Comm. 18 (1976) 19.

[6] I.V. Tomov, R. Fedosejevs and M.C. Richardson, Appl. Phys. Lett. 30 (1977) 164

[7] I.V. Tomov, R. Fedosejevs and M.C. Richardson, Optics Comm. 21 (1977) 327.

[8] W. Seka and J. Bunkenburg, J. Appl. Phys. 49 (1978) 2277.

[9] I.V. Tomov, R. Fedosejevs and M.C. Richardson, Rev. Sci. Instrum. 50 (1979) 9.

[10] G.F. Albrecht and J. Bunkenburg, Optics Comm. 38 (1981) 377.

[11] L.S. Goldberg and P.E. Schoen, IEEE J. QE-20 (1984) 628.

[12] P. Heinz, W. Kriegleder and A. Laubereau, Appl. Phys. A43 (1987) 209.

[13] G.V. Krivoshchekov, N.G. Nikulin, and V.A. Smirnov, Sov. J. Quant. Electron. 5 (1976) 1096.

[14] H.P. Kortz, IEEE J. QE-19 (1983) 578.

[15] Y.C. Yao and Z.G. Zhang, Appl. Phys. B40 (1986) 157.

[16] L.A. Lompre, G. Mainfray and J. Thebault, J. Appl. Phys. 48 (1977) 1570.

[17] R.J. Collins and P. Kisliuk, J. Appl. Phys. 33 (1962) 2009.

[18] W. Koechner, Solid-state laser engineering (Springer-Verlag, New York, 1976).

[19] J. Sapriel, Acousto-optics (Wiley, Chichester, 1979).

[20] G.F. Albrecht, L. Lund and D. Smith, Appl. Optics 22 (1983) 1276.

[21] D.R. Preuss and J.L. Gole, Appl. Optics 19 (1980) 702

[22] A.E. Siegman, IEEE J. QE-9 (1973) 247.

[23] R. Trutna and A.E. Siegman, IEEE J. QE-13 (1977) 955.

[24] H. Vanherzeele, J.L. Van Eck, and A.E. Siegman, Appl. Optics 20 (1981) 3484.

[25] D. von der Linde and K.F. Rodgers, IEEE J. QE-9 (1973) 960.

[26] F. Graf and A. Penzkofer, Opt. Quant. Electorn. 17 (1985) 53.

[27] IntraAction data sheet.

[28] A. Penzkofer, D. von der Linde and A. Laubereau, Optics Comm. 4 (1972) 377.

[29] J.A. Giordmaine, P.M. Rentzepis, S.L. Shapiro and K.W. Wecht, Appl. Phys. Lett. 11 (1967) 216.

[30] A. Penzkofer and W. Leupacher, J. Luminesc. 37 (1987) 61.

[31 ] O.G. Peterson, J.P. Webb, J.C. McColgin and J.E. Eberly, J. Appl. Phys. 42 (1971) 1917.

[32] Schott data sheets.

[33] R.C. Jones, J. Opt. Soc. Am. 31 (1941) 488

[34] American Institute of Physics Handbook, ed. D.E. Gray (McGraw Hill, New York, 1972) p. 6-27. 